\title{
Sistema Classificador de Pavimentação Utilizando Acelerômetros e Máquina de Vetores de Suporte
}

\author{
Filipe do Ó Cavalcanti, Waslon T. A. Lopes e Fabrício B. S. de Carvalho
}

\begin{abstract}
Resumo-Este artigo apresenta o desenvolvimento de um sistema robusto para classificação de pavimentação, fazendo uso de uma Máquina de Vetores de Suporte (SVM - Support Vector Machine) e o processamento de dados obtidos a partir de acelerômetros montados em um veículo. Os dados obtidos em um trajeto urbano foram separados em segmentos e destes foram extraídas métricas, utilizadas no treinamento da SVM. O sistema foi capaz de classificar os dados entre asfalto ou paralelepípedos com $97 \%$ de precisão.
\end{abstract}

Palavras-Chave-Máquina de Vetores de Suporte, Acelerômetros, Aprendizado de Máquina, Pavimentação.

Abstract-This paper describes the design of a robust pavement classifier, utilizing a Support Vector Machine (SVM) and processed data from accelerometers mounted to a vehicle. Data was obtained in a urban scenario and separated into segments, from which some metrics were extracted and used to train the SVM. This system was able to classify data between asphalt or paving stone with $97 \%$ accuracy.

Keywords-Support Vector Machine, Accelerometers, Machine Learning, Pavement.

\section{INTRODUÇÃO}

Desde a inserção dos veículos automotores no mercado, algumas mudanças foram permanentes na dinâmica das cidades. Bairros e novos zoneamentos passaram a ser definidos primeiramente por suas ruas, em que o carro tem um importante papel. No entanto, a qualidade das ruas e avenidas pode diferir bastante dentro de um bairro. É comum avenidas e ruas principais possuírem construção em asfalto, enquanto ruas secundárias (aquelas que apenas possuem imóveis residenciais, em geral) têm sua construção com alvenaria poliédrica, também chamada de construção em paralelepípedos [1].

Naturalmente, trechos asfaltados caracterizam uma maior fluidez no trânsito, pois permitem uma circulação em maior velocidade e oferecem mais conforto ao motorista e passageiros. As ruas em paralelepípedo oferecem menor conforto devido às suas características físicas, consequentemente menor velocidade. No entanto, apresenta como vantagem um menor custo de construção [1]. As diferenças citadas entre os dois tipos de pavimentos são facilmente perceptíveis para os motoristas devido à sensação de trepidação no veículo.

O uso de acelerômetros para caracterizar vibrações em veículos é algo comum. Em [2] foi instalado um acelerômetro

Filipe do Ó Cavalcanti, Waslon T. A. Lopes e Fabrício B. S. de Carvalho, Programa de Pós-Graduação em Engenharia Elétrica, Universidade Federal da Paraíba (UFPB), João Pessoa-PB e GCOMPS (Grupo de Pesquisa em Comunicações e Processamento de Sinais). Os autores agradecem o apoio da UFPB e do Conselho Nacional de Desenvolvimento Científico Tecnológico - CNPq (CNPq No. 315514/2018-3) a esta pesquisa. E-mails: filipe.cavalcanti@cear.ufpb.br,waslon@cear.ufpb.br, fabricio@cear.ufpb.br. no para-brisas para detecção de irregularidades na pista, e utiliza esta informação para determinar desbalanceamento nos pneus. Uma outra maneira de abordar o uso de acelerômetros foi com o uso da plataforma de desenvolvimento Arduino, capturando dados a uma frequência de amostragem de $2 \mathrm{~Hz}$ e aplicando o cálculo de desvio padrão para classificar a qualidade da pavimentação [3]. A aquisição das informações em uma frequência maior se mostra necessária quando a velocidade do veículo e a sensibilidade do acelerômetro são levadas em consideração. Utilizando uma taxa de $40 \mathrm{~Hz}$ e o acelerômetro fixado no painel de instrumentos, o sistema apresentado em [4] analisa os valores médio, de pico e valor quadrático médio (RMS - Root Mean Square) da aceleração para analisar os efeitos das vibrações no corpo humano. Essas informações são relevantes se analisadas de maneira apropriada, podendo ser utilizadas para classificar a qualidade de ruas em relação a anomalias presentes (buracos, bueiros, etc).

Em [5] os autores propõem um sistema capaz de armazenar dados de acelerômetros que foram instalados próximos às rodas e no painel de instrumentos, além de obter entradas do usuário (que informam o tipo de anomalia na rua). A aquisição não teve uma taxa fixa devido a problemas no sistema montado. Os dados são separados em três grupos de análise e treinam uma Árvore de Classificação e Regressão que foi capaz de classificar a qualidade das ruas em 4 níveis distintos.

Neste contexto, este trabalho propõe uma abordagem mais robusta do ponto de vista da montagem do sistema de aquisição, desenvolvendo um sistema capaz de obter dados de um acelerômetro de três eixos instalado no chassi e próximo ao centro de gravidade do veículo, garantindo que as vibrações do veículo sejam devidamente transmitidas ao módulo de aquisição. As aquisições de dados foram realizadas a uma frequência constante de $100 \mathrm{~Hz}$ com resolução de 16 bits. As informações adquiridas são processadas por conjuntos de 100 amostras, gerando métricas bem definidas que serão utilizadas para treinar uma Máquina de Vetores de Suporte (SVM Support Vector Machine) e, por fim, classificar os dados entre asfalto ou alvenaria poliédrica.

O restante deste trabalho está organizado da seguinte maneira: na Seção II é apresentada uma breve introdução sobre SVM. Na Seção III é descrita a montagem física dos acelerômetros ao veículo e dados referentes ao sistema eletrônico utilizado para aquisição de dados. Na Seção IV, define-se o que são as métricas de análise e quais foram extraídas a partir do domínio do tempo e da frequência. A Seção V explica como os dados foram tratados e escolhidos para treinar a SVM. Por 
fim, as Seções VI e VII relatam os resultados obtidos e as conclusões, respectivamente.

\section{Máquina De Vetores de Suporte}

Máquina de Vetores de Suporte é uma técnica de classificação em que vetores de entrada são mapeados de maneira não-linear em um espaço de dimensões superiores. Neste espaço é construída uma superfície de decisão linear, que representa a maior margem entre os vetores de duas classes, chamada de hiperplano [6]. Quando os dados de entrada são facilmente separáveis, o hiperplano será uma reta que contém a maior distância (margem) entre duas categorias. Quando esta separação não é possível em duas dimensões, aplica-se um mapeamento aos dados que irá elevar a dimensão do problema. Em três dimensões o hiperplano passa a ser um plano, como mostrado na Figura 1, onde duas classes distintas usadas de exemplo são representadas pelas cores azul e laranja.

Três kernels (ou núcleos) são normalmente utilizados na literatura: linear, radial e polinomial. Para todos os núcleos, dois parâmetros devem ser escolhidos: C e gamma. C é responsável pela relação entre simplicidade da região de decisão, ou seja, o quanto as classificações podem errar durante o treinamento (C muito alto pode levar a overfitting ou sobreajuste). Gamma indica a área de influência dos pontos de treinamento. Se for muito pequeno, pode não acompanhar a "forma" dos dados corretamente. Porém, se for muito grande, irá se restringir aos próprios pontos de treinamento. Portanto, é necessário testar valores de $\mathrm{C}$ e gamma para encontrar o que melhor se adapta aos dados.

Este tipo de aprendizado de máquina é muito eficaz quando os dados estão claramente separados e também quando o número de categorias é maior que o número de amostras. No entanto, dados com ruído irão reduzir a eficácia e, caso a quantidade informação de entrada seja excessiva, o tempo de processamento irá elevar com o cubo do volume de dados.

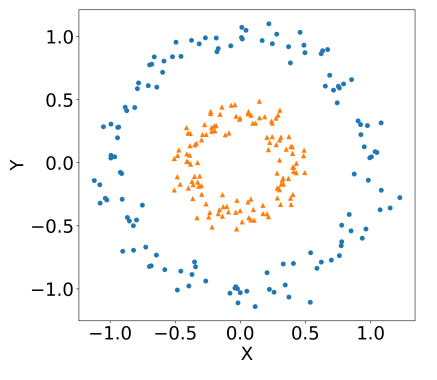

(a) Visualização em $R^{2}$.

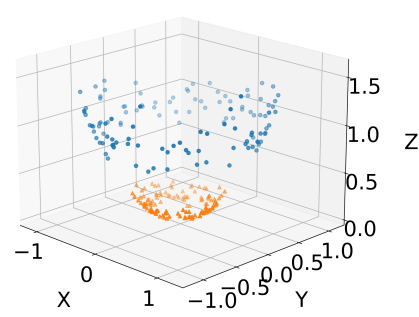

(b) Visualização em $R^{3}$.
Fig. 1: Visualização do mapeamento de $R^{2}$ para $R^{3}$.

SVM podem ser aplicadas para classificação nas mais diversas áreas. Devido a crise sanitária global causada pelo novo Coronavírus (Sars-CoV-2), pesquisadores observaram que o diagnóstico para COVID-19 poderia ser executado com mais rapidez que os testes normais, a partir de imagens de raio-x do pulmão. Foram utilizadas técnicas de extração de características de imagens combinados com máquinas de vetores de suporte para obter até $99,18 \%$ de acurácia para classificação entre pulmões saudáveis, com pneumonia ou com COVID-19 [7].

Além de classificação de características de imagem, SVM também são utilizadas para classificação de texto, sendo capaz de identificar a informação em e-mails quanto à presença ou não de spam [8], atuando como filtro na caixa de entrada do usuário.

A classificação de dados em função do tempo também é abordada por SVM. [8] faz uso de múltiplos sensores instalados nos braços de um grupo de participantes, que fornecem sinais a partir do movimentos das mãos. As características dos sinais foram classificados utilizando SVM, obtendo uma acurácia significante em relação a outros métodos de classificação.

\section{DESCRIÇÃO DO SISTEMA}

No sistema proposto, o módulo acelerômetro é acoplado ao chassi do veículo, mais especificamente ao trilho do banco do passageiro. Este local foi escolhido devido à sua proximidade do centro de gravidade do veículo e por ser um local ideal para análises relacionadas à dirigibilidade, uma vez que as vibrações neste local irão representar com fidelidade as vibrações transmitidas ao motorista. Conforme mostrado na Figura 2, a montagem do acelerômetro é feita ao trilho do banco de maneira que os eixos $X, Y$ e $Z$ do acelerômetro estejam alinhados com os eixos longitudinal, lateral e vertical do veículo, respectivamente.

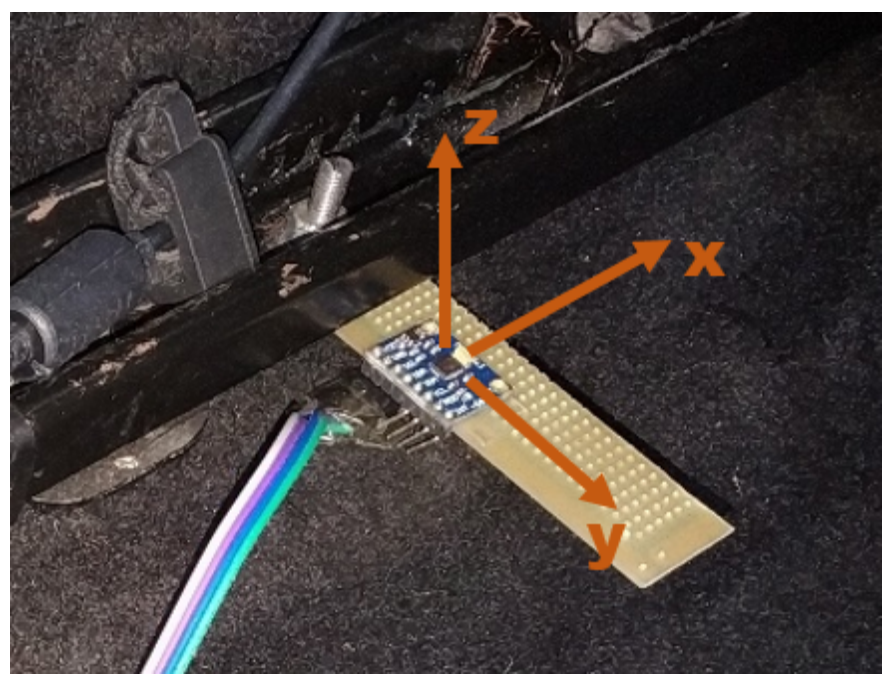

Fig. 2: Instalação do acelerômetro ao trilho do banco.

Além do alinhamento dos eixos, a instalação deve ser feita de maneira que o plano $X Y$ esteja montado o mais paralelo ao solo possível, evitando que a força gravitacional exerça influência nos eixos $X$ e $Y$.

O módulo utilizado foi o MPU6050 produzido pela TDK InvenSense [9], que dispõe de um conversor analógico-digital (ADC) de 16 bits para amostragem de dados do acelerômetro, sendo configurado para uma sensibilidade de $+/-2 \mathrm{~g}(1 \mathrm{~g}=$ $\left.9,8 \mathrm{~m} / \mathrm{s}^{2}\right)$ e amostrado a uma frequência de $100 \mathrm{~Hz}$. A comunicação é feita via interface $\mathrm{I}^{2} \mathrm{C}$ a partir da placa de desenvolvimento Tiva-C [10], que conta com um processador ARM Cortex-M4 de 32 bits operando a $120 \mathrm{MHz}$. Os dados 
dos três eixos são enviados em tempo real para um computador utilizando comunicação UART e salvos em um arquivo no formato de texto tabulado (.txt).

\section{MÉtricAs de ANÁlise}

A partir dos dados originais (em que AccX, AccY e AccZ serão utilizados para referenciar a aceleração dos eixos $X, Y$ e $Z$, respectivamente), é aplicado um filtro de média móvel com uma janela de 5 amostras em AccZ, gerando uma nova coluna de dados AccZ_RA5, sendo esta nova série representativa de AccZ filtrado de altas frequências.

Em seguida, todo o conjunto de dados é separado em segmentos que possuem 100 amostras (ou 1 segundo de dados da aquisição). De cada um desses segmentos serão extraídas métricas que servirão para o treinamento da técnica de aprendizado supervisionado. Foram extraídas métricas em dois domínios diferentes: tempo e frequência.

\section{A. Métricas de Análise no Tempo}

Cinco métricas foram geradas utilizando os dados amostrados no tempo. Duas dessas métricas envolvem os três eixos para serem calculadas. A primeira é a aceleração média do intervalo, que indica a amplitude média da aceleração quando visualizada em forma vetorial [11]. A aceleração média no intervalo para um eixo é definida como:

$$
x_{\text {med }}=\frac{1}{M} \sum_{i=1}^{M} x_{(i-1)},
$$

sendo

$$
k=1,2, \ldots, M \text {. }
$$

em que $M$ é o número de amostras do segmento. Aplica-se a Equação 1 para os demais eixos (AccY e AccZ) e por fim, a aceleração média, $a c e l_{m e d}$, é dada por

$$
\text { acel }_{\text {med }}=\sqrt{x_{\text {med }}^{2}+y_{\text {med }}^{2}+z_{\text {med }}^{2}} .
$$

A segunda métrica de análise no tempo é a aceleração RMS no intervalo, que fornece uma indicação da potência média do sinal no tempo devido apenas às fontes de aceleração oscilatórias [11]. A aceleração RMS do segmento em um eixo é

$$
x_{r m s}=\sqrt{\frac{1}{M} \sum_{i=1}^{M} x_{i-1}^{2}},
$$

sendo

$$
k=1,2, \ldots, M \text {. }
$$

A Equação 3 é aplicada aos eixos restantes e a magnitude do vetor aceleração RMS é:

$$
\text { acel }_{r m s}=\sqrt{x_{r m s}^{2}+y_{r m s}^{2}+z_{r m s}^{2}} .
$$

As três outras métricas são o desvio padrão em AccX (X_Stddev), desvio padrão em AccZ (Z_Stddev) e a última métrica é a maior variação pico-a-pico da coluna de dados AccZ_RA5 (Z_Pk2pk) no segmento. A Figura 3 exibe as métricas calculadas dos segmentos 240 até 370 de uma aquisição (os dados exibidos foram filtrados para melhor visualização)
Nota-se que asfalto e paralelepípedo apresentam amplitudes claramente distintas para cada métrica, com exceção de Acc_Mean e Acc_RMS em certos pontos, que apresentam picos em momentos em que o veículo está executando uma curva. Este pico é significativo devido à variação da aceleração lateral do veículo representada por AccY. Os valores que representam a variável Terreno estão detalhados na Tabela I.

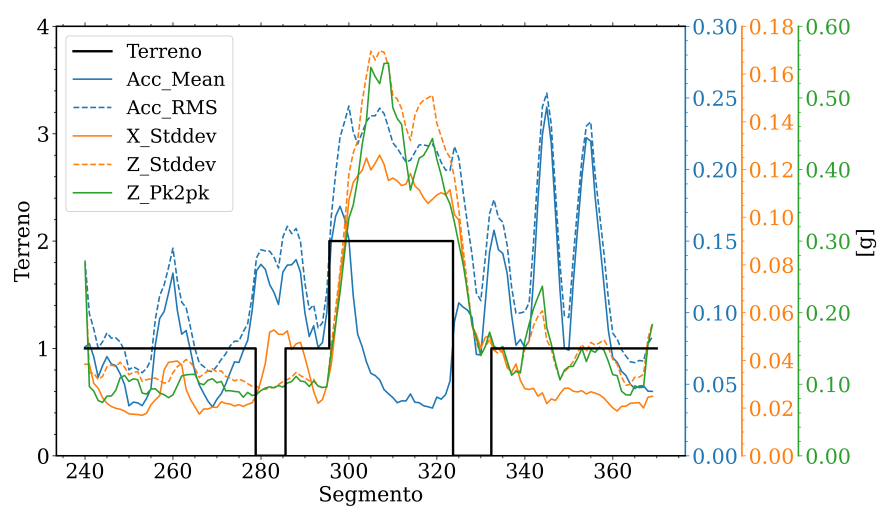

Fig. 3: Visualização das métricas no domínio do tempo.

TABELA I: Representação numérica do terreno.

\begin{tabular}{|l|c|c|c|c|c|}
\hline Terreno & Indefinido & Asfalto & Paralelepípedo & Barro & Lombada \\
\hline Valor & 0 & 1 & 2 & 3 & 4 \\
\hline
\end{tabular}

\section{B. Métricas de Análise em Frequência}

A análise em frequência por meio da Transformada Rápida de Fourier (FFT - Fast Fourier Transform) permite extrair informações preciosas de um segmento. Sabendo que a frequência de amostragem do sinal é de $100 \mathrm{~Hz}$, a FFT irá fornecer informações até $50 \mathrm{~Hz}$. Três métricas são extraídas deste domínio, todas relativas ao eixo $\mathrm{Z}$.

A primeira métrica calcula a média de todas as amplitudes obtidas ao calcular a FFT do segmento (Z_FFT_Mean). A segunda e terceira métricas calculam as médias das amplitudes de diferentes regiões. Z_Low_Freq representa a média das amplitudes consideradas de baixa frequência, que foram definidas como sendo a faixa de 1 a $10 \mathrm{~Hz}$. Z_High_Freq irá calcular a média com valores considerados de alta frequência, na faixa de 35 a $50 \mathrm{~Hz}$. A visualização das métricas descritas pode ser observada na Figura 4, sob o mesmo intervalo de dados utilizado na Figura 3.

\section{Processamento dos Dados}

Durante o experimento foram gerados 2096 segmentos, o que equivale a aproximadamente 35 minutos de aquisição de dados em pavimentações de asfalto ou paralelepípedo. Todos os segmentos em que o veículo estava parado ou sobre uma lombada foram filtrados, portanto a análise a seguir irá considerar apenas os trechos asfaltados ou com paralelepípedo, resultando em 1980 segmentos O processamento foi executado em Python, utilizando a biblioteca scikit-learn para todo o trabalho relacionado a SVM. 


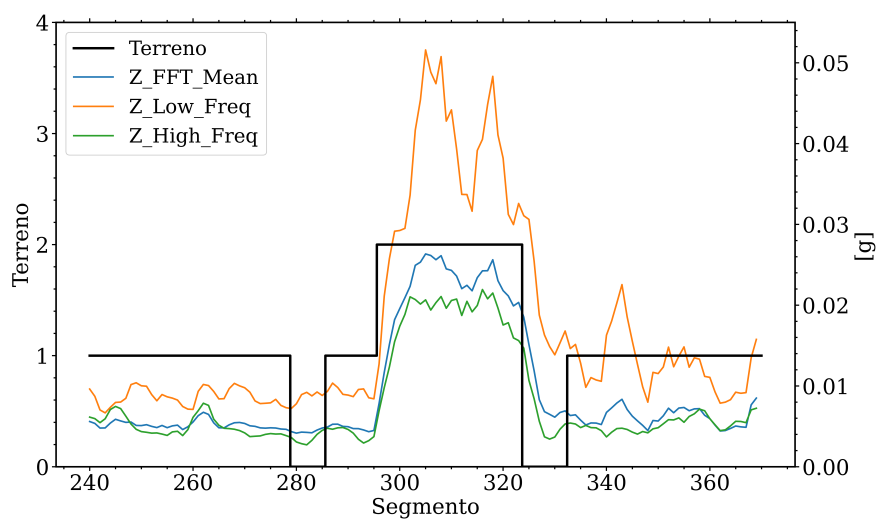

Fig. 4: Visualização das métricas no domínio da frequência.

Previamente ao uso de técnicas de aprendizado de máquina, os dados devem ser normalizados. Foi utilizada a função StandardScaler para remover a média e manipular os dados para obter variância unitária. Esta função normalizou todas as métricas extraídas dos segmentos e, a partir destes valores, foi calculada a correlação entre os dados. A Figura 5 mostra a correlação entre as oito métricas calculadas, em que cores mais claras indicam maior correlação e cores mais escuras, menor correlação.

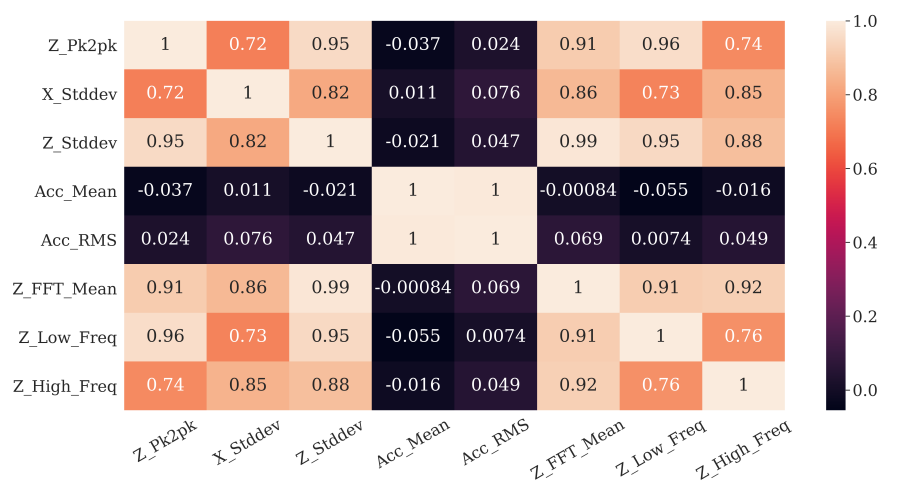

Fig. 5: Correlação entre as métricas calculadas.

A partir da Figura 5, nota-se que os valores de correlação para Acc_Mean e Acc_RMS são iguais. Isto ocorre devido à redundância de informação presente nos valores RMS e médio.

Fica claro que as métricas Z_Pk2pk, Z_Stddev, Z_FFT_Mean e Z_Low_Freq possuem as melhores correlações, com valores superior a 0,9. Por isso, estas quatro métricas foram escolhidas para o treinamento da SVM.

\section{RESUltados}

Para este trabalho, a SVM irá trabalhar em quatro dimensões (devido às quatro métricas escolhidas). 2100 amostras (equivalentes a 35 minutos de aquisição de dados) foram separados em uma proporção $80 \%$ e $20 \%$, respectivamente. Para avaliar qual a melhor configuração da SVM, foram testadas 48 combinações de parâmetros, sendo permutados três tipos de núcleo (linear, radial e polinomial), quatro valores de gamma e quatro valores de C. A configuração que entregou o melhor resultado utilizou núcleo radial, gamma igual a 0,1 e C igual a 10. Os resultados das permutações podem ser visualizados na Figura 6.

Utilizando o conjunto de testes, composto por 420 segmentos (equivalentes a sete minutos de aquisição com o carro em movimento), foram obtidos acertos em 97\% dos 286 segmentos em asfalto e $98 \%$ para 134 segmentos em pavimentação de alvenaria poliédrica. A Tabela II expõe as principais métricas de classificação.

TABELA II: Métricas de avaliação da SVM.

\begin{tabular}{|r|c|c|c|c|}
\hline & Precisão & Revocação & F1-Score & Elementos \\
\hline Asfalto & $97 \%$ & $99 \%$ & $98 \%$ & 286 \\
\hline Paralelepípedo & $98 \%$ & $93 \%$ & $95 \%$ & 134 \\
\hline Média & $97 \%$ & $96 \%$ & $97 \%$ & 420 \\
\hline
\end{tabular}

As métricas da Tabela II são calculadas em relação às predições de cada elemento, que podem ser: verdadeiro positivo, falso positivo, verdadeiro negativo e falso negativo. Precisão calcula a proporção de classificações positivas corretas (verdadeiro positivo) em relação aos casos que realmente são corretos (verdadeiros positivos e falsos negativos). Revocação indica a taxa de acertos verdadeiros positivos em relação aos verdadeiros positivos e falsos negativos. A métrica F1-Score combina revocação e precisão em uma média harmônica.

Ambas as classes apresentam resultados satisfatórios na precisão. A revocação para classificação de pavimentação poliédrica possui um valor inferior a média, o que pode ser atribuído a trechos de asfalto de má qualidade que foram classificados como falsos negativos.

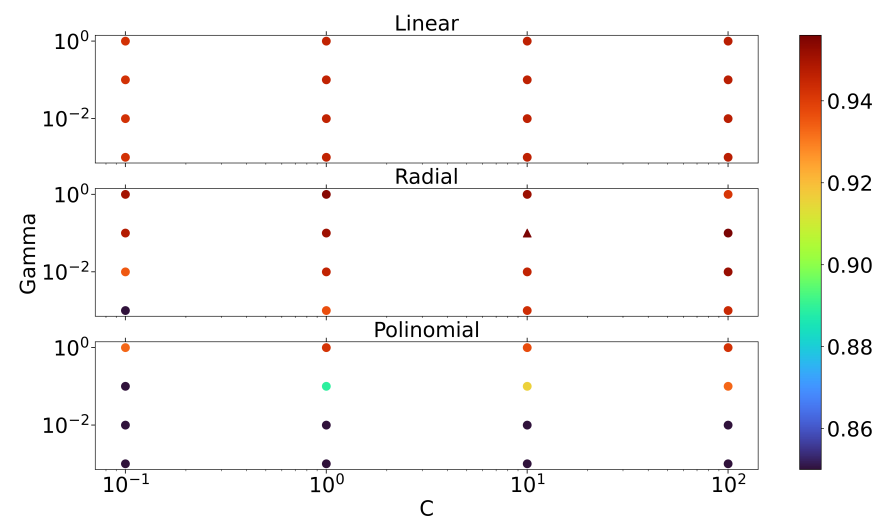

Fig. 6: Precisão para valores de C e Gamma.

\section{CONCLUSÕES}

Este trabalho apresentou a implementação de um sistema capaz de classificar o tipo de pavimentação a partir do uso de acelerômetros. Utilizando um microcontrolador e um módulo acelerômetro de três eixos, foi possível obter dados com alta taxa de precisão e com alta taxa de aquisição. A aplicação de métricas simples extraídas do domínio do tempo e da frequência mostrou-se efetiva, sendo capaz de treinar uma SVM para classificar os segmentos entre duas classes (asfalto e paralelepípedo) com precisão de $97 \%$.

Como continuação deste trabalho, serão aplicadas outras técnicas de aprendizado de máquina visando definir qual tem 
a maior precisão com a menor complexidade. Uma segunda abordagem será utilizar as métricas para classificar a qualidade das ruas e utilizar geolocalização para que os locais que se destaquem sejam apontados em um mapa.

\section{REFERENCIAS}

[1] M. B. Megier, J. R. Hammarstron, B. M. de Quevedo, R. D. Palmeira, and B. F. Finckler, "Análise comparativa de pavimento asfáltico, pavimento em alvenaria poliédrica e pavimento intertravado em bloco de concreto," XXVI Seminário de Iniciação Científica Unijuí, 2018.

[2] K. Prażnowski and J. Mamala, "Classification of the road surface condition on the basis of vibrations of the sprung mass in a passenger car," IOP Conf. Series: Materials Science and Engineering, 2016.

[3] S. B. Prapulla, S. N. Rao, and V. A. Herur, "Road quality analysis and mapping for faster and safer travel," International Conference on Energy, Communication, Data Analytics and Soft Computing (ICECDS), 2017.

[4] P. Raghavendra, M. Sachin, P. S. Srinivas, and V. Talasila, "Three dimensional road quality measurement and analysis," International Conference on Circuits, Communication, Control and Computing, 2014.

[5] H. Tariq, S. Mazhar, and H. Hammed, "Poster abstract: Road quality classification for road repair authorities and regular drivers, using an on-board data logger," 17th ACM/IEEE International Conference on Information Processing in Sensor Networks (IPSN), 2018.

[6] C. Cortes and V. Vapnik, "Support-vector networks," Machine Learning, 1995.

[7] M. Turkoglu, "Covidetectionet: Covid-19 diagnosis system based on $\mathrm{X}$-ray images using features selected from pre-learned deep features ensemble," Applied Intelligence, 2021.

[8] H. Zeng, K. Li, X. Tian, N. Wei, R. Song, and L. Zhou, "Classification of hand motions using linear discriminant analysis and support vector machine," in Chinese Automation Congress (CAC), pp. 2353-2356, 2017.

[9] T. InvenSense, "MPU-6050 MEMS MotionTracking Device." https: //invensense.tdk.com/products/motion-tracking/ 6-axis/mpu-6500/. Acessado em: Abril de 2021.

[10] T. Instruments, "EK-TM4C1294XL." https://www.ti.com/ tool/EK-TM4C1294XL. Acessado em: Abril de 2021.

[11] M. J. B. Rogers, K. Hrovat, K. McPherson, M. E. Moskowitz, and T. Reckart, "Accelerometer data analysis and presentation techniques," tech. rep., Tal-Cut Company and NASA Lewis Research Center, 1997. 